\title{
Philosophiques
}

\section{Les dangers d'une Charte des droits enchâssée pour un Québec indépendant}

\section{Josée Legault}

Volume 19, numéro 2, automne 1992

Une nation peut-elle se donner la constitution de son choix?

URI : https://id.erudit.org/iderudit/027196ar

DOI : https://doi.org/10.7202/027196ar

Aller au sommaire du numéro

Éditeur(s)

Société de philosophie du Québec

ISSN

0316-2923 (imprimé)

1492-1391 (numérique)

Découvrir la revue

Citer ce document

Legault, J. (1992). Les dangers d'une Charte des droits enchâssée pour un Québec indépendant. Philosophiques, 19(2), 145-155.

https://doi.org/10.7202/027196ar d'utilisation que vous pouvez consulter en ligne.

https://apropos.erudit.org/fr/usagers/politique-dutilisation/ 


\title{
LES DANGERS D'UNE
}

\section{CHARTE DES DROITS ENCHÂSSÉE \\ POUR UN QUÉBEC INDÉPENDANT}

\author{
par \\ Josée Legault
}

De nos jours, tenter de discuter des dangers inhérents à l'enchâssement d'une Charte des droits n'est guère facile. En fait, cela peut devenir parfois carrement dangereux. Il demeure encore mal vu et considéré comme inconvenable de remettre en question la sagesse du principe même d'une Charte des droits enchâsseee, que nous parlions d'un Québec indépendant ou non. Une telle position amène souvent des accusations de tendance fascisante, d'intolérance et d'anti-libéralisme. Et pourtant, le texte qui suit est loin de constituer la première tentative au Canada d'offrir une analyse critique de telles chartes. Par exemple, le juriste Michael Mandel, collègue de Peter Hogg et professeur au Osgoode School of Law, publiait, en I989, une analyse non moins critique des effets politiques de la Charte canadienne, dans son fameux The Charter of Rights and the Legalization of Politics in Canada.

Depuis l'enchâssement de la Charte canadienne, suite au « coup de force » fédéral de I982, une littėrature critique - mais discrète - కest peu à peu constituée dans les périodiques de droit et de science politique. Tout récemment, François Rocher et Daniel Salée, respectivement de l'Université Carleton et Concordia, publiaient dans le numéro 20 de la Revue québécoise de science politique, un article intitulé "Charte et socièté: vers un 
nouvel ordre canadien $»$, dans lequel ils reprenaient les grandes lignes de l'analyse de Michael Mandel, et concluaient que cette Charte était fort loin de constituer une avancée politique importante. Force est toutefois de constater que nous, les critiques, demeurons surpassés en nombre et en influence par les intellectuels « procharte » qui en clament les vertus démocratiques.

Beaucoup d'encre a coulé sur, et à propos de la Charte canadienne, nonobstant le fait que le Québec s'était doté d'une Charte à forte tendance individualiste et ce, dès i975. Cible des critiques et symbole de la tendance centralisatrice et égalitariste trudeauiste, la Charte canadienne a réussi à éclipser son homologue québécoise, et les intellectuels tendent souvent, non seulement à en oublier l'existence, mais également à oublier qu'advenant l'indépendance du Québec, une Charte des droits enchâssée comporterait certes des risques semblables à celle d'Ottawa. C'est à cette question que nous tenterons de réfléchir.

Nous nous proposons de réflèchir sur certains des effets pervers qu'aura eu la Charte canadienne, parce qu'elle est enchâssée, et fait appel à ce que l'on nomme le « judicial review », c'est-à-dire l'interprétation de la Charte par des juges non élus. Il va sans dire que la liste des principaux effets pervers d'une Charte enchâssée, telle que proposée ici, demeure non exhaustive.

Quant à la Charte québécoise, quoiqu'elle jouisse d'un statut supérieur aux autres lois de l'Assemblée nationale, elle demeure soumise à la Charte canadienne, loi suprême du pays. Voilà pourquoi nous aborderons les effets pervers de la charte canadienne, pendant qu'en filigrane, nous y verrons poindre les effets tout aussi potentiellement pervers d'une charte québécoise enchâssée dans un Québec indépendant.

Mais avant de procéder à la démonstration, quelques mots seront consacrés à certains facteurs ayant motivé le choix d'un sujet aussi délicat. En fait, quatre facteurs nous ont amené à prendre position publiquement sur cette question, qui demeure, nous désirons le préciser en toute modestie, hors de notre champ immédiat de spécialisation.

Brève liste des facteurs de motivation:

I. Plusieurs années de réflexion solitaire, extrêmement solitaire, sur cette question depuis l'enchâssement de la 
Charte canadienne (nous étions trop jeune pour saisir les implications de la Charte québécoise...).

2. De longues, très longues conversations que nous avons eu l'immense plaisir d'avoir depuis une année avec le professeur Gary Caldwell, premier intellectuel avec qui nous partagions les mêmes craintes et questionnements.

3. Le mot d'un représentant anglo-québécois, prononcé récemment lors d'une des conférences constitutionnelles de Monsieur Clark, et selon qui l'on se devait d'abolir le plus rapidement possible les clauses dèrogatoires des chartes québécoise et canadienne, puisque cela permettrait enfin aux deux gouvernements de se réclamer de l'infaillibilité de la charte afin de justifier l'impossibilité d'y contrevenir (ex: affichage commercial).

4. Les interventions périodiques d'intellectuels québécois affirmant la supériorite de la Charte québécoise sur sa soeur canadienne, dont le professeur Guy Laforest, qui écrivait récemment:

I...l la première condition d'un véritable partenariat entre le Canada et le Quẻbec (est que) le Québec doit instaurer sur son territoire la primauté de sa propre Charte des droits interpretée par des juges nommés par les autorités gouvernementales québécoises.

Et ce, dans le même paragraphe où le professeur Laforest notait pourtant la « centralisation du système judiciaire », de même que l'« immense liberté interprétative des tribunaux » que la Charte imposait au processus démocratique...

A ce moment, nous nous sommes dit qu'il était grand temps de coucher sur papier certains des questionnements qui nous assaillaient depuis dejjà trop longtemps. Que cela contribue ou non, d'ailleurs, à ce que nous appelons tous, de manière un peu prétentieuse, la connaissance.

\section{EFFET PERVERS N" l: LE GOUVERNEMENT DES JUGES OU IES DANGERS DU « JUDICIAL REVIEW 》}

Au Canada, on ne sait trop comment ni pourquoi, depuis I982, les tribunaux et les juges semblent sêtre appropriés des qualités rares qui feraient, de toute évidence, défaut aux divers parlements: celles de l'objectivité, de l'impartialité, bref du nonarbitraire. La Cour suprême, tel le sage perché au haut de sa montagne, serait donc habilitée à rendre des décisions d'une portée considérable pour les diverses sociétés composant le Canada. La vague rumeur qui circulait depuis longtemps en Occident sur l'indépendance et la suprématie morale de la « justice » (dans un 
système où, paradoxalement, les juges sont, de toute façon, nommés par le Premier ministre du Canada), semble avoir pris depuis 1982, des proportions mythiques, d'où, entre autres, la réaction virulente contre l'utilisation de la clause dérogatoire en décembre 1988.

En effet, comment un gouvernement osait-il se soustraire à la décision rendue par le plus haut tribunal du pays, veritable parole d'évangile, dont on ne pouvait douter qu'au prix d'être accusé d'hérésie ou de sacrilège politique, à moins que ce ne soit la sempiternelle accusation de « fascisme » et d $\mathrm{d}$ antidèmocratisme »...?

Cette tendance à glorifier et sanctifier des juges absolument et irrevocablement non imputables face à une population dépourvue de mécanismes de révocation (rôle admirablement joué par les élections dans le cas des parlements), laisse songeur quant à la conception qu'ont de plus en plus de Canadiens et de Québécois de ce qu'est ou devrait être la démocratie. Une telle forme de démocratie - celle des juges et de l'interprétation juridique - n'est ni directe, ni représentative, ni parlementaire, ni présidentielle, ni socialiste, ni libérale. En fait, elle va à l'encontre de l'essence même de la démocratie. Tout devient alors une question de perception lune décision rendue étant perçue comme « juste » par certains et « injuste » par d'autres), l'important étant que l'imputabilité ou la responsabilité face à la population demeure, dans ce cas-ci, inexistante.

Ce vice, dont souffre de manière congènitale une Charte des droits enchâssée, est par définition extrêmement difficile à amender et, fonctionnant sur la base de ce qu'il est donné d'appeler le « judicial review », une Charte québécoise enchâssée interprétée par des juges nommés par les autorités gouvernementales québécoises (pour emprunter le mot de Guy Laforest), en souffrirait tout autant.

Nous savons que cette confiance envers le processus de «judicial review » découle d'une crainte légitime de l'« arbitraire » que l'on prête de plus en plus aux parlements et du besoin que ressentent certains citoyens et intellectuels de s'assurer que des contrôles extra-parlementaires existent.

Qu'en est-il vraiment de ces inquiétudes? Mis à part le fait que les parlements sont, de par leur imputabilité et responsabilité, beaucoup moins susceptibles à l'arbitraire que des juges non élus qui n'ont jamais à répondre de leurs décisions, précisons que dans le système prèsent, les juges sont, de toute façon, 
nommés par le Premier ministre du Canada (dans un Québec indépendant, ils seraient, sans aucun doute, nommés par le Premier ministre de cet Etat). Peut-on vraiment croire qu'un Premier ministre désirant faire infléchir les décisions rendues d'un côté particulier ne pourrait le faire en nommant des juges qui partagent sa « vision des choses »?... Dans un pareil cas, les cours risquent de rendre des décisions plutôt conformes à celles qui auraient été rendues, de toute façon, par le pouvoir politique!

Dans le cas contraire, où un Premier ministre nommerait ses juges de manière "impartiale », il faudrait alors s'attendre à la possibilité que ceux-ci rendent des décisions allant à l'encontre de celles prises par le pouvoir politique, et ce, nous le répétons, sans avoir à en rendre compte à la ou aux populations concernées.

D̛un côté, nous avons un travesti d'impartialité et d'objectivité, et de l'autre, un pouvoir parallèle mais supérieur au pouvoir politique, parce que de derniere instance et non imputable. Mais certains diront, qu'un peu comme les roses, l'important, c'est la clause ... dérogatoire. Alors là, il faut savoir ce que l'on veut. Ou bien l'on fait confiance aux parlements et on les soumet au jugement de la population tous les quatre ans, ou bien on ne leur fait pas confiance, et on leur impose une charte des droits enchâssée où les juges ont le pouvoir de contester les décisions prises par ces mêmes parlements. Dans ce dernier cas, cependant, il n'est pas question de clause dérogatoire. Une cohérence minimale, au niveau des principes et de l'application des contrôles dits extra-parlementaires est tout de même requise de la part de ceux qui défendent une Charte des droits enchâssée. Ce minimum de cohérence exige, de leur part, la répudiation de la clause dérogatoire.

Quant à la souveraineté parlementaire avec responsabilité, elle se doit d'être réelle - mais non absolue, tout « absolutisme» étant contraire au principe de la démocratie - ou elle se doit d'être soumise aux tribunaux. Tout comme une femme ne saurait être enceinte à moitié, le parlement ne peut être souverain à moitié, ou pis encore, seulement lorsque cela ferait l'affaire du Premier ministre. Encore une fois, une cohérence minimale semble de mise ici.

\section{EFFET PERVERS N 2: UNE CHARTE DES DROITS ENCHÂSSÉE} ET LA LANGUE aU QUÉbeC

Ceux qui auront lu, au complet, les arrêts rendus sur les contestations portées devant les tribunaux contre certaines 
sections de la Charte de la langue française (loi Ior), dont le plus célèbre demeure celui ayant mené à l'adoption en Ig88, de la loi I78 sur l'affichage commercial, en savent déjà long sur la supposée impartialité des juges.

En effet, ces décisions furent, plus souvent qu'autrement, et qui saurait s'en ètonner, de nature politique. Les juges ont ainsi eu le pouvoir de décider de ce que le Québec avait besoin, ou non, pour protéger ce que l'on nomme avec grande sollicitude, sa « spécificité » ou son caractère « distinct », lequel est réduit à la langue française, et encore, selon des balises imposées par une autorité fédérale, soit la cour suprême.

Encore une fois, ce ne fut pas à des représentants élus mais à des juges non élus que l'on confia cette tâche aussi lourde et importante. Le résultat, celui que l'on connaît dans le cas de la langue de l'affichage commercial, nous ramène de nouveau à l'incohèrence évidente d'une Charte des droits enchâssée munie d'une clause dérogatoire.

Mais cela n'est que la pointe de l'iceberg, et encore, de l'iceberg fédéral. Car la Charte québécoise, en ce qui concerne la question de la langue, est loin d'être dépourvue de dents aptes à déchiqueter ce qui reste de la loi ror, dans l'éventualité où elle serait enchâssée dans la loi suprême du Québec indépendant. Nous en voulons pour preuve les articles 3 et ro sur lesquels les cours se sont basées pour invalider certaines sections de la loi IOI, et ce, bien avant la Charte canadienne de 1982. En effet, et cela paraît difficile à croire, la Charte québécoise, contrairement à celle du fédéral, interdit toute discrimination basée sur la langue (art. Io). Ce qui, au Quèbec, n'a aucun sens. Encore une fois, dans un Québec indépendant, une fois une telle Charte enchâssée, ou le gouvernement respectera celle-ci, et l'on peut envisager la possibilité, par exemple, de retourner au libre choix de la langue d'enseignement (la Charte canadienne en délimitant, au moins minimalement, les critères d'accès à l'article 23), ou le gouvernement conservera sa clause dérogatoire et devra l'utiliser de manière systématique avec le prix politique qui s'ensuivrait inévitablement.

Pour ce qui est de la possibilité de se doter d'une version québécoise du fameux article i de la Charte canadienne sur les limites justes et raisonnables dans une société libre et démocratique (autre règle interprétative) - version qui existe déjà timidement à l'article g.r de la Charte québécoise - nous conseillons à quiconque entrevoit cette possibilité de lire, au complet, ce que 
les juristes appellent communément le « test Oakes », lequel dicte les règles d'application de l'article i de la Charte canadienne. Ce " test », que l'on applique afin de déterminer si une loi ou un règlement contrevenant à la charte peut tout de même constituer une limite jugée juste et raisonnable dans une société libre et démocratique, et qui fait appel, entre autres choses, à d'obscurs et complexes critères des proportionnalité et de liens dits rationnels; la lecture de ce test finirait de convaincre n'importe quel politologue ou philosophe des dangers inhérents à la remise de tels pouvoirs décisionnels entre les mains de juges et d'avocats

Car le test Oakes, d'une importance pourtant cruciale en ce qui a trait à l'application concrète de la Charte, demeure totalement inaccessible non seulement au commun des mortels, c'està-dire aux électeurs, de par sa complexité impressionnante, mais en toute probabilité à la très grande majorité des politologues québécois et canadiens. Cela est, c'est le moins que l'on puisse dire, inquiétant.

En fait, dans un Québec indépendant, il serait préférable de réécrire la loi ıor dans le sens où nous l'exposons dans le livre L'invention d'une minorité. Les Anglo-Québécois ${ }^{1}$, C'est-à-dire en réunissant en une seule section tout ce qui concerne la minorité anglophone, plutôt que de risquer des interprétations basées sur la Charte, lesquelles iraient inévitablement à l'encontre de l'esprit et de la lettre de la loi ror. De plus, dans un Québec indépendant, il y aurait sans aucun doute une mouvance migratoire non négligeable dans les premières années, et il sera alors d'une importance cruciale de se doter d'instruments législatifs souples afin de $s$ adapter aux nouvelles circonstances qui risquent d'apparaître. Face à une situation démolinguistique potentiellement volatile, on voit tout de suite apparaître ici un sous-effet pervers, soit la réduction substantielle de notre capacité d'adaptation, danger qu'une constitution minimaliste sans Charte enchâssée ne court pas.

\section{EFFET PERVERS N $N^{\prime}$ : CENTRALISATION ET TENDANCE À L'ÉGALITARISME}

La Charte canadienne, ce n'est plus un secret pour personne, a facilité la centralisation du pouvoir judiciaire et politique. En remettant entre les mains d'une Cour dite suprême le pouvoir

I. Josée Legault, L'invention d'une minorité. Les Anglo-québécois, Montréal, Boréal, I992. 
décisionnel de dernière instance en des matières d'une importance cruciale pour la population entière, elle centralise l'exercice même du pouvoir, tout en en arrachant des pans entiers au Parlement.

Dans un Québec indépendant, doté d'une structure constitutionnelle semblable à celle du Canada - et ici, l'ironie n'est pas involontaire - l'effet serait le même.

La nouvelle Cour suprême du Québec deviendrait ainsi l'instrument privilégié d'une centralisation et d'une égalitarisation qui ne respecterait plus la diversité sociologique, économique, politique, etc., des nombreuses régions et populations qui composeraient ce nouvel État. Avec ou sans indépendance, c'est d'une décentralisation importante dont le Québec a un urgent besoin, et non le contraire. Lors du présent Colloque, nous entendions une des présentatrices nous dire combien la Charte canadienne ouvrait les portes à la diversité. La discussion passionnée qui s'en est suivie nous fit voir à quel point il est loin d'y avoir unanimité sur cette question.

\section{EFFET PERVERS N $N^{2}$ 4: RÉDUCTION DE I'ACCÈs À LA JUSTICE}

Une Charte des droits enchâssée, baseee sur le principe de l'interprétation juridique et possédant un pouvoir décisionnel de dernière instance, coûte cher. Elle coûte cher à l'État, en frais de représentation (les tribunaux étant devenus d'énormes trous à fonds publics), mais elle coûte surtout cher à ses citoyens désirant y faire appel. Le processus de contestation juridique via une Charte des droits est, dans la plupart des cas, extrêmement onéreux et peut prendre de nombreuses années. Seuls les groupes ou individus possédant la capacité de payer et d'attendre peuvent, dans les faits, s'en prévaloir. À moins que l'État décide d'en assumer la totalité des frais, auquel cas il finance l'érosion de sa propre souveraineté. Sinon, il laisse les citoyens seuls face aux requins et technocrates du droit.

\section{EFFET PERVERS N" 5: CRÉATION D'UNE CUITURE JURIDIQUE ÉVACUANT LA CULTURE POLITIQUE}

Une Charte des droits enchâssée avec interprétation juridique change le sens même du droit, puisque la justice n'intervient plus seulement pour sanctionner les citoyens ayant transgressé un interdit contenu dans une législation particulière, elle dicte également ce qui est permis et ce qui doit être dans une sociètè. Ce qui relevait du consensus sociétal, des traditions, des coutumes, du non-dit, du non-écrit, de la 
négociation et même parfois des confrontations, est maintenant dicté par des textes juridiques, soit explicites lc'est-à-dire «coulé dans le bèton »), soit interprètatif (c'est-à-dire relevant des juges non élus).

La culture politique du citoyen sen retrouve inévitablement atrophiée, et la dynamique sociétale entre les individus et les groupes s'en trouve également altérée de manière importante.

A plus long terme, une Charte peut avoir l'effet de produire un abandon dangereux (et souvent inconscient) des concepts de concertation et de médiation entre citoyens et groupes. L'avocat et le juriste deviennent ainsi les nouveaux et principaux médiateurs entre l'État et le citoyen. Le résultat inévitable est celui d'un éloignement accentué du citoyen par rapport à l'Etat, voire son isolement. En bout de ligne, le seul appui qui demeure pour le citoyen - dans la mesure où il peut se payer ses services demeure son ou ses avocats.

On assiste alors à une dépolitisation de la société civile, laquelle ne fait que sadapter à ses propres gouvernants qui, abandonnant leurs responsabilités, préfèrent de plus en plus travestir les questions politiques importantes en questions purement juridiques.

\section{EFFET PERVERS N* 6: EVACUATION DE}

\section{LA NOTION DE POUVOIR ET DE IUTTES DE POUVOIR}

En réduisant les relations entre individus et groupes à une simple, et oserions-nous dire « simpliste », question de «droits », une Charte enchâssée évacue du discours politique et sociologique jusqu'à la notion même de pouvoir et, surtout, de luttes de pouvoir. Deux brefs exemples: les Anglo-Québécois, qui avec leur rhétorique basée sur les droits individuels, réussissent à faire oublier qu'il est question ici de droits et intérêts collectifs et de lutte de pouvoirs entre deux collectivités, et, bien entendu, les autochtones, qui acceptent de parler du « droit » inhérent à lautonomie gouvernementale plutôt que du « pouvoir » de se gouverner eux-mêmes.

Là-dessus, Michael Mandel demeure celui ayant fait la démonstration la plus convaincante de cette aseptisation artificielle des conflits inter-individus et inter-groupes. En remplaçant les notions de pouvoir, de luttes de pouvoir et de relations conflictuelles par la notion transcendantale de «droits», d'autres effets pervers apparaissent. 
Par exemple, le discours public et individuel tend à opérer un glissement inquiétant en substituant au discours de la responsabilité, de la participation et de la démocratie, celui des droits, plaçant le citoyen et les groupes dans une situation attentiste, transformant ceux-ci en simples « quémandeurs » de droits. On voit poindre lentement une population de plus en plus incapable de réfléchir en termes de responsabilités, voire d'identifier le concept même de responsabilité. Tandis que celui de " justice », pourtant si fondamental, se voit également remplacé par celui des «droits ».

\section{Conclusion}

Une Charte des droits québécoise enchâssee et soumise à l'interprétation juridique comporterait certes des dangers et des effets pervers semblables à ceux de la Charte canadienne. En fait, si la Charte d'Ottawa est un mythe fondateur d'une «nation» canadienne déconnectée de ce qu'est en fait, ce pays, il est à craindre qu'une Charte québécoise enfante un nationalisme tout aussi déconnecté de ce qu'est, et pourrait devenir, un Québec independant.

Que l'on parle de la création extrêmement dispendieuse et dangereuse de cette nouvelle caste de médiateurs que sont les avocats, de la judiciarisation des questions politiques, de l'instauration d'un gouvernement de juges non élus et donc, non imputables, de l'incompatibilité d'une telle Charte avec l'esprit et la lettre d'une législation du type de la loi Ior, de la tendance centralisatrice et égalitariste, de la réduction de l'accès à la justice, de l'évacuation de la culture politique du citoyen, de l'aseptisation artificielle des conflits sociaux, ou de la menace directe à la société civile et à la relation qu'elle se doit d'entretenir avec l'Etat, on ne peut plus se défiler devant l'obligation pour les intellectuels québécois de réflèchir sérieusement aux implications d'une telle Charte pour un Québec independant.

Doit-on se croiser les bras devant cet État appelé à devenir un gigantesque administrateur de «droits »? Ou doit-on trouver des moyens de rendre la démocratie plus efficace, de la renforcer plutôt que de l'affaiblir?

Les constitutions et les chartes doivent être au service du citoyen. Cela peut paraître naïf, mais des principes de ce type devront, à notre humble avis, retrouver leurs lettres de noblesse dans la réflexion des intellectuels. Après tout, ce n'est pas au citoyen de se mettre au service de sa Constitution ou de sa Charte. Le patriotisme constitutionnel d'Habermas, dont une 
présentatrice nous parle dans le cadre de ce Colloque, n'a, dans une telle perspective, que bien peu de sens.

L'auteure tient toutefois à souligner que si le principe de souveraineté parlementaire lui semble préférable à celui d'interprétation juridique, elle ne nie d'aucune façon le caractère hautement « perfectible » du système parlementaire d'inspiration britannique qu'un Québec indépendant risque fort de conserver. Mais seulement dans la mesure où la principale prémisse à la base d'une réforme de ce système est le renforcement de la démocratie et la réhabilitation de la société civile. Dans un tel contexte, le principe d'une Charte des droits enchâssée avec interpretation juridique, tout comme celui d'un pouvoir exécutif trop puissant, sont à exclure.

Dans le cadre spècifique de l'indépendance du Québec, une question fondamentale pour la qualité de la vie démocratique de ce nouvel Etat doit être posée. En effet, faire un pays, se séparer afin de se retrouver dans le même bourbier constitutionnel et chartiste que celui du Canada, en vaut-il vraiment la peine? Car, si une nation, ou dans ce cas-ci, un nouvel ttat constitué à même l'amputation d'un autre, peut se donner la constitution de son choix, le moindre qu'il doit aux citoyens qui endureront le sacrifice de la séparation, c'est de ne pas répéter les erreurs commises par l'Etat canadien qu'il vient de quitter.

Voilà pourquoi en cette année du roe anniversaire de la Charte canadienne, et alors que la charte québécoise approche déjà de ses vingt ans, nous devons confronter le fait qu'elles soient ici devenues l'avatar de la société idéale.

De quelle société idéale, demanderez-vous? Mais celle de Pierre Elliott Trudeau, bien entendu, le même qui parcourt maintenant le monde et tente de vendre aux Ukrainiens et aux SudAfricains l'idée d'une Charte des droits enchâssée avec interprétation juridique. L'art d'exporter les èchecs...

En effet, comment ne pas s'exclamer de concert avec le journaliste Pierre Nadeau, qui faisait état, en ces termes, des derniers voyages du pèlerin de l'Avenue des Pins:

Pauvres Ukrainiens, ils n'auront jamais suffisamment de lacs pour noyer les chagrins qu'ils se préparent. Quoique, après tout, ils aient bien une Mer noire!... »

Département de sciences politiques (doctorat)

Université du Québec à Montréal 